\title{
Downlink MIMO HCNs with Residual Transceiver Hardware Impairments
}

\author{
Anastasios Papazafeiropoulos and Tharm Ratnarajah,
}

\begin{abstract}
A major limitation of heterogeneous cellular networks (HCNs) is the neglect of the additive residual transceiver hardware impairments (ARTHIs). The assumption of perfect hardware is quite strong and results in misleading conclusions. This paper models a general multiple-input multiple-output (MIMO) HCN with cell association by incorporating the RTHIs. We derive the coverage probability and shed light on the impact of the ARTHIs, when various transmission methods are applied. As the hardware quality decreases, the coverage probability worsens. Especially, this effect is more severe as the transmit power increases. Furthermore, we verify that in an $\mathrm{HCN}$, it is better to employ at each base station as few transmit antennas as possible.
\end{abstract}

Index Terms-Heterogeneous cellular network, MIMO systems,transceiver hardware impairments, stochastic geometry, coverage probability.

\section{INTRODUCTION}

Recently, heterogeneous cellular networks (HCNs) have attracted a significant interest for 5 th generation $(5 \mathrm{G})$ wireless systems [1]. The maturity of HCNs, started from single-input single-output (SISO) links [2], has enabled the coexistence of multiple-antenna strategies [3], [4].

Over the years, there has been an increasing focus on investigating the effects of transceiver hardware impairments (THIs) on the performance of wireless communication systems such as phase noise [5], high power amplifier nonlinearities [6], Inphase/Quadrature-phase (I/Q)-imbalance [7]. Although calibrations schemes at the transmitter and compensation algorithms at the receiver exist, their efficacy is limited, since a certain amount of inevitable residual impairments still remains due to several reasons, e.g., the time-variation of the hardware characteristics. Thus, the additive residual THIs (RTHIs), modeling the aggregate effect of all the residual transceiver impairments, arise [8]-[13]. Despite that HCNs are a candidate solution for $5 \mathrm{G}$ systems [1], no evaluation of the impact of the ARTHIs has taken place regarding HCNs in the literature.

In this paper, we make a step beyond [3] and [4], which considered ideal hardware, in order to assess the effect of the ARTHIs on HCNs. Specifically, we consider a downlink multiple-input multiple-output (MIMO) $\mathrm{HCN}$ in the presence of the RTHIs. Moreover, we derive the coverage probability in terms of tools from stochastic geometry. The result enables us to illustrate the impact of the ARTHIs on the performance of HCNs and draw a picture on their effects on the multipleantenna transmission strategies.

\section{SySTEM MODEL}

In this paper, we consider a cellular MU-MISO system, having one BS per cell, drawn according to an independent

A. Papazafeiropoulos and T. Ratnarajah are with the Institute for Digital Communications (IDCOM), University of Edinburgh, Edinburgh, EH9 3JL, U.K., (email: a.papazafeiropoulos, t.ratnarajah@ed.ac.uk).

This work was supported by the U.K. Engineering and Physical Sciences Research Council (EPSRC) under grant EP/L025299/1.
Poisson Point Process (PPP) $\Phi_{B}$ with density $\lambda_{B}$. Each BS deploys a large number of antennas $M$ that is greater or equal to the number of associated users $K$, i.e., $M \geq K$. Also, the user locations are modeled by an independent PPP $\Phi_{u}$ with density $\lambda_{u}=6 \lambda_{B}$. Moreover, the same time-frequency resource is shared by the users across all cells. Slivnyak's theorem allows conducting the analysis by focusing on a typical user found at the origin. We assume that the users belong to the Voronoi cell of the nearest BS, and the set of all the cells comprise a Voronoi tessellation.

\section{A. Downlink Transmission}

We assume that the desired channel power from the BS located at $x \in \mathbb{R}^{2}$ to the typical user, found in its cell, is given by $h_{k}$, while the inter-cell interference power from another BS (located at $y_{l} \in \mathbb{R}^{2}$ ) is denoted by $g_{y}$.

Assuming knowledge of perfect channel state information (CSI) at the transmitter side, we focus on ZF precoding. Hence, the received signal from the $j$ th $\mathrm{BS}$ to user $k$ at $x$ in its cell, after applying the ZF precoder, can be expressed as

$$
y_{k}=\mathbf{h}_{k}^{\mathrm{H}} \mathbf{s}_{k}\|x\|^{-\alpha / 2}+\sum_{l \in \Phi_{B} / x} \mathbf{g}_{l}^{\mathrm{H}} \mathbf{s}_{l}\left\|y_{l}\right\|^{-\alpha / 2}+n_{k},
$$

where $\mathbf{s}_{k}=\mathbf{W}_{k} \mathbf{d}_{k} \in \mathbb{C}^{M \times 1}$ is the transmit signal vector for the $k$ th user with covariance matrix $\mathbf{Q}=\mathbb{E}\left[\mathbf{s}_{k} \mathbf{s}_{k}^{\mathrm{H}}\right]$ and $p_{k}=\operatorname{tr}(\mathbf{Q})$ is the associated average transmit power. In particular, we assume that the linear precoding is denoted by the matrix $\mathbf{W}_{k} \in \mathbb{C}^{M \times K}$, employed by the BS, which multiplies the data signal vector $\mathbf{d}_{k}=\left[d_{k, 1}, d_{k, 2}, \cdots, d_{k, K}\right]^{\top} \in \mathbb{C}^{K} \sim$ $\mathcal{C N}\left(\underline{0}, \mathbf{I}_{K}\right)$ for all users in that cell. Also, $\alpha$ is the pathloss exponent parameter. The channel vectors $\mathbf{h}_{k} \in \mathbb{C}^{M \times 1}$ and $\mathbf{g}_{l} \in \mathbb{C}^{M \times 1}$ denote the desired and interference channel vectors between BSs located at $x \in \mathbb{R}^{2}$ and $y_{l} \in \mathbb{R}^{2}$ far from the typical user. In the case of Rayleigh fading, the channel power distributions of both the direct and the interfering links follow the Gamma distribution [14]. Also, $\mathbf{n}_{k}$ is an additive white Gaussian noise (AWGN) vector, such that $n_{k} \sim \mathcal{C N}(0,1)$.

In practice, both the users and the BSs are affected by certain inevitable residual additive impairments [8], [15]. Given the channel realizations, the conditional transmitter and receiver distortion noises for the $i$ th link are modeled as Gaussian distributed, where their average power is proportional to the average signal power, as shown by measurement results [8]. In other words, we have]

$$
\begin{aligned}
\boldsymbol{\eta}_{\mathrm{t}} & \sim \mathcal{C N}\left(\underline{0}, \delta_{\mathrm{t}}^{2} \operatorname{diag}\left(q_{1}, \ldots, q_{M}\right)\right) \\
\eta_{\mathrm{r}} & \sim \mathcal{C N}\left(\underline{0}, \delta_{\mathrm{r}}^{2}\|x\|^{-\alpha} \mathbf{h}_{k}^{\mathrm{H}} \operatorname{tr}(\mathbf{Q}) \mathbf{h}_{k}\right)
\end{aligned}
$$

\footnotetext{
${ }^{1}$ We assume that all the BSs have the same hardware impairments without any loss of generality.
} 
where $q_{1}, \ldots, q_{M}$ are the diagonal elements of $\mathbf{Q}$. Note that the circularly-symmetric complex Gaussianity can be justified by the aggregate contribution of many impairments. The proportionality parameters $\delta_{\mathrm{t}}^{2}$ and $\delta_{\mathrm{r}}^{2}$ describe the severity of the residual impairments at the transmitter and the receiver side. In applications, these parameters are met as the error vector magnitudes (EVM) at each transceiver side [16].

Remark 1: The receive distortion includes the path-loss coming from the associated $\mathrm{BS}^{2}$.

Hence, the hardware impairments are written as $\boldsymbol{\eta}_{\mathrm{t}} \sim$ $\mathcal{C N}\left(\underline{0}, p_{k} \delta_{\mathrm{t}}^{2} \mathbf{I}_{K}\right)$ and $\eta_{\mathrm{r}} \sim \mathcal{C} \mathcal{N}\left(\underline{0}, p_{k} \delta_{\mathrm{r}}^{2}\|x\|^{-\alpha}\left\|\mathbf{h}_{k}\right\|^{2}\right)$.

Incorporating these parameters to (1), we obtain

$$
y_{k}=p_{k} \mathbf{h}_{k}^{\mathrm{H}}\left(\mathbf{s}_{k}+\boldsymbol{\eta}_{\mathrm{t}}\right)\|x\|^{-\frac{\alpha}{2}}+\sum_{l \in \Phi_{B} / x} p_{l} \mathbf{g}_{l}^{\mathrm{H}} \mathbf{s}_{l}\left\|y_{l}\right\|^{-\frac{\alpha}{2}}+\eta_{\mathrm{r}}+n_{k} .
$$

Proposition 1: The signal-to-interference (SIR) ratio of the downlink transmission from the BS to its typical user, accounting for transceiver hardware impairments, in an $\mathrm{HCN}$ can be represented by

$$
\gamma_{k}=\frac{p_{k} h_{k}\|x\|^{-\alpha}}{I_{\boldsymbol{\eta}_{\mathrm{t}}}\|x\|^{-\alpha}+I_{l}+I_{\boldsymbol{\eta}_{\mathrm{r}}}\|x\|^{-\alpha}},
$$

where $h_{k}=\left|\mathbf{h}_{k} \mathbf{w}_{k, k}\right|^{2} \sim \Gamma\left(\Delta_{k}, 1\right)$, and $\Delta_{k}=M-K+1$. Note that $\mathbf{w}_{k, k}$ is the $k$ th column of $\mathbf{W}_{k}$. In addition, we have $I_{\boldsymbol{\eta}_{\mathrm{t}}} \sim p_{k} \delta_{\mathrm{t}}^{2} \Gamma(M, 1)$, and $I_{\boldsymbol{\eta}_{\mathrm{r}}} \sim p_{k} \delta_{\mathrm{r}}^{2} \Gamma(M, 1)$ while the total interference from all the other base stations found at a distance $\|y\|$ from the typical user is $I_{l}=\sum_{l \in \Phi_{B} / x} p_{l} g_{l}\|y\|^{-\alpha}$, where $g_{l} \sim \Gamma(K, 1)$.

Proof: See Appendix A

\section{Coverage Probability}

This section, starting with a formal definition of the coverage probability with BS locations drawn from a PPP, presents the technical derivation of an upper bound of the downlink coverage probability of a typical user in a MIMO HCN. While different transmission techniques are employed that depend on the number of BS antennas $M$ and the number of users in each cell $K$, the inherent existence of residual additive transceiver hardware impairments is incorporated in the analysis.

The generality of the model allows the investigation of the effects of hardware imperfections on the coverage probability towards a more realistic assessment.

Definition 1 ([4]): A typical user is in coverage if its effective downlink SIR from at least one of the randomly located BSs in the network is higher than the corresponding target. In general, we have 3

$$
p_{c}=\mathbb{E}\left[\mathbb{1}\left(\underset{x \in \Phi_{B}}{\cup} \operatorname{SIR}(\mathrm{x})>\mathrm{T}\right)\right],
$$

where the indicator function $\mathbb{1}(e)$ is 1 when event $e$ holds and 0 otherwise.

The following theorem is the main result, being unique in the research area of practical systems with hardware impairments, when the BSs are randomly positioned. It is based on

\footnotetext{
${ }^{2}$ Note that the ARTHIs from other BSs are negligible due to the increased path-loss. Also, the transmit hardware impairment depends only on the transmit signal power from the tagged BS and not from the path-loss.

${ }^{3} \mathrm{We}$ assume that the thermal noise is negligible as compared to the distortion noises and the other cells interference as showed by simulations. However, it can be included in the proposed analysis by means of some extra work.
}

the calculation of the Laplace transforms provided by means of Proposition 2 and Lemma 1.

Theorem 1: The downlink probability of coverage $p_{c}\left(T, \lambda_{B}, \alpha, \delta_{\mathrm{t}}, \delta_{\mathrm{r}}\right)$ in a general cellular network with randomly distributed multiple-antenna BSs, accounting for additive transceiver hardware impairments, is given by

$$
\begin{gathered}
p_{c}\left(T, \lambda_{B}, \alpha, \delta_{\mathrm{t}}, \delta_{\mathrm{r}}\right) \leq \lambda_{B} \int_{l \in \mathbb{R}^{2}} \sum_{i=0}^{\Delta-1} \sum_{k=0}^{i} \sum_{n=0}^{i-k}\left(\begin{array}{c}
i \\
k
\end{array}\right)\left(\begin{array}{c}
i-k \\
n
\end{array}\right) \\
\times \frac{(-1)^{i} \tilde{T}^{i-k} s^{k}}{i !} \frac{\mathrm{d}^{n}}{\mathrm{~d} s^{n}} \mathcal{L}_{I_{\eta_{\mathrm{r}}}}(s) \frac{\mathrm{d}^{i-k-n}}{\mathrm{~d} s^{i-k-n}} \mathcal{L}_{I_{\eta_{\mathrm{t}}}}(s) \frac{\mathrm{d}^{k}}{\mathrm{~d} s^{k}} \mathcal{L}_{I_{l}}(s) \mathrm{d} l,
\end{gathered}
$$

where $l=\|x\|, s=\tilde{T} l^{a}$, and $\tilde{T}=T p_{k}^{-1}$, while $\mathcal{L}_{I_{\eta_{\mathrm{r}}}}(s)$, $\mathcal{L}_{I_{\eta_{\mathrm{t}}}}(s)$, and $\mathcal{L}_{I_{l}}(s)$ are the Laplace transforms of the powers of the receive distortion, transmit distortion, and interference power coming from other BSs.

Proof: See Appendix B.

Remark 2: In the ideal case of no ARTHIs, (6) coincides with the coverage probability provided by Theorem 3 in [4] for single tier.

Proposition 2: The Laplace transform of the interference power of a general cellular network with randomly distributed multiple-antenna BSs having additive transceiver hardware impairments is given by

$$
\mathcal{L}_{I_{l}}(s)=\exp \left(-s^{\frac{2}{a}} \mathcal{C}(\alpha, K)\right),
$$

where $\mathcal{C}(\alpha, K)=\frac{2 \pi \lambda_{B}}{a} \sum_{m=0}^{K}\left(\begin{array}{l}K \\ m\end{array}\right) \mathrm{B}\left(K-m+\frac{2}{a}, m-\frac{2}{a}\right)$.

Proof: See Appendix C

Lemma 1: The Laplace transforms of the parts, describing the ARTHIs $I_{\eta_{\mathrm{t}}}$ and $I_{\boldsymbol{\eta}_{\mathrm{r}}}$, are given by

$$
\mathcal{L}_{I_{\eta_{j}}}(s)=\frac{1}{\left(1+q_{j} s\right)^{M}},
$$

where $j=\mathrm{t}$ or $\mathrm{r}$ and $q_{t}=\delta_{\mathrm{t}}^{2}$ or $q_{r}=\delta_{\mathrm{r}}^{2}$, respectively.

Proof: Both Laplace transforms are easily obtained, since $I_{\eta_{\mathrm{t}}}$ and $I_{\boldsymbol{\eta}_{\mathrm{r}}}$ follow scaled gamma distributions with scaled parameters $p_{k} \delta_{\mathrm{t}}^{2}$ and $p_{k} \delta_{\mathrm{r}}^{2}$, as mentioned in Appendix $\mathrm{A}$.

The numerical evaluation of (6) is complex and timeconsuming because it involves the calculation of the derivatives of Laplace transforms.

Remark 3: In the general case, where $\Delta>1$, the derivatives of the Laplace transforms, being composite functions, are calculated by applying Faà di Bruno's identity. In the case of $\mathcal{L}_{I_{l}}(s)$, if we denote the composite function as $(f \circ g)(s)$, then $f(s)=\exp (s)$ and $g(s)=-\tilde{s}^{\frac{2}{a}} \mathcal{C}(\alpha, \mathcal{M})$. Similarly, in the case of $\mathcal{L}_{I_{\boldsymbol{\eta}_{j}}}(s)$, we have $f(s)=\frac{1}{s^{M}}$ and $g(s)=1+q_{j}^{2} s$.

Corollary 1: In the special case of full space division multiple access (SDMA) $(M=K)$, the upper bound of the coverage probability with residual transceiver impairments, described by Theorem 1, is given by

$$
p_{c}\left(T, \lambda_{B}, \alpha, \delta_{\mathrm{t}}, \delta_{\mathrm{r}}\right) \leq \lambda_{B} \int_{x \in \mathbb{R}^{2}} \mathcal{L}_{I_{\boldsymbol{\eta}_{\mathrm{r}}}}(s) \mathcal{L}_{I_{\boldsymbol{\eta}_{\mathrm{t}}}}(s) \mathcal{L}_{I_{l}}(s) \mathrm{d} x .
$$

\section{NumericAl RESUltS}

The locations of BSs are simulated as realizations of a PPP with given density in a sufficiently large window of $5 \mathrm{~km} \times 6 \mathrm{~km}$. Moreover, we assume that the typical user lies at the origin, and we calculate the desired signal strength and the interference power. The coverage probability is obtained by checking if the received SIR from at least one of the 
BSs is more than the target value. The "solid" and "dot" lines designate the analytical results with no ARTHIs and specific ARTHIs, respectively, while the bullets represent the simulation results.

In Fig. 1, the simulated coverage probability $p_{c}$ along with the proposed analytical result (6) are plotted against the target SIR $T$ for $\delta_{t}=0.15$ and $\delta_{r}=04$. These nominal values of ARTHIs are quite reasonable according to [10]. Moreover, in the same figure, we have depicted the simulated and theoretical results corresponding to ideal hardware as provided by (21) in [4]. Obviously, in practice, where ARTHIs exist, $p_{c}$ worsens as lower hardware quality is used (increasing $\delta_{t}, \delta_{r}$ ). In addition, we consider three different transmission strategies. In particular, we have i) single-user beamforming (SU-BF) with $M=6, K=1$, ii) $M=K=1$, i.e, each BS has a single transmit antenna (SISO), and iii) $M=K=6$, which means full SDMA. Similar to [4] we show that SU-BF transmission is preferable with comparison to SISO, while the latter is better than SDMA. In other words, we verify that it is better to serve a single user in each resource block, either by SISO or SU$\mathrm{BF}$, instead of serving multiple users. However, herein and with comparison to [4], we illustrate how, given this property, the coverage probability varies with SIR in the presence of the ARTHIs.

SU-BF case has an additional beamforming gain;

Fig. 2 further illustrates the effect of increasing the transmit BS power. This exposes a quite insightful property, since the ARTHIs are power-dependent. Specifically, increasing the ARTHIs, the space $l_{i}$ for $i=1,2$, representing the gap between the lines with no hardware impairments and the practical scenario with ARTHIs, becomes larger, i.e., $l_{1}>l_{2}$ for $\rho=15 \mathrm{~dB}$ and $\rho=5 \mathrm{~dB}$, respectively.

\section{CONCLUSION}

Contrary to existing works on HCNs, assuming ideal hardware, this paper studied the impact of the ARTHIs. In particular, based on a general realistic scenario, where a BS can employ several multiple-antennas downlink transmission strategies after taking into account for cell association, we obtained the coverage probability in the presence of the unavoidable ARTHIs. It was showed that the ARTHIs degrade the coverage capability. More importantly, it was showed that this degradation is higher as the transmit power increases.

\section{APPENDIX A}

\section{ProOF OF PROPOSITION 1}

We assume that the columns of the precoding matrix $\mathbf{W}_{k}$ equal the normalized columns of $\mathbf{H}^{\mathrm{H}}\left(\mathbf{H H}^{\mathrm{H}}\right)^{-1}$, i.e., $\mathbf{W}_{k}=$ $\overline{\mathbf{H}}^{\mathrm{H}}\left(\overline{\mathbf{H}} \overline{\mathbf{H}}^{\mathrm{H}}\right)^{-1}$, where $\overline{\mathbf{H}}=\left[\overline{\mathbf{h}}_{1}, \ldots, \overline{\mathbf{h}}_{k}\right]^{\mathrm{H}} \in \mathbb{C}^{(K \times M)}$ with

\footnotetext{
${ }^{4}$ Note that based on the proposed model the transmit and receive additive impairments have equivalent effect, although it should be stressed that in reality the BS's transmitter and user's receiver are manufactured with different quality.

${ }^{5}$ In interference-limited networks, the claim having more antennas is always beneficial is not necessarily correct, as it heavily depends on how the transmit antennas are used and which transmission/reception scheme is employed. For instance, using the transmit antennas for multi-stream transmission (SDMA) is not beneficial (from a system perspective) in most cases (with treating interference as noise). Furthermore, the correctness of the claim depends on the performance metric we study. In general, there are regimes/situations where SU-BF is better than SISO. The main reason why SU-BF can outperform SISO is that in addition to the proximity gain enjoyed by the SISO due to extreme densification, the SU-BF transmission presents an additional beamforming gain. Also, we should take into account that the growth of the received signal and interference for increasing $\lambda_{B}$ is the same.
}

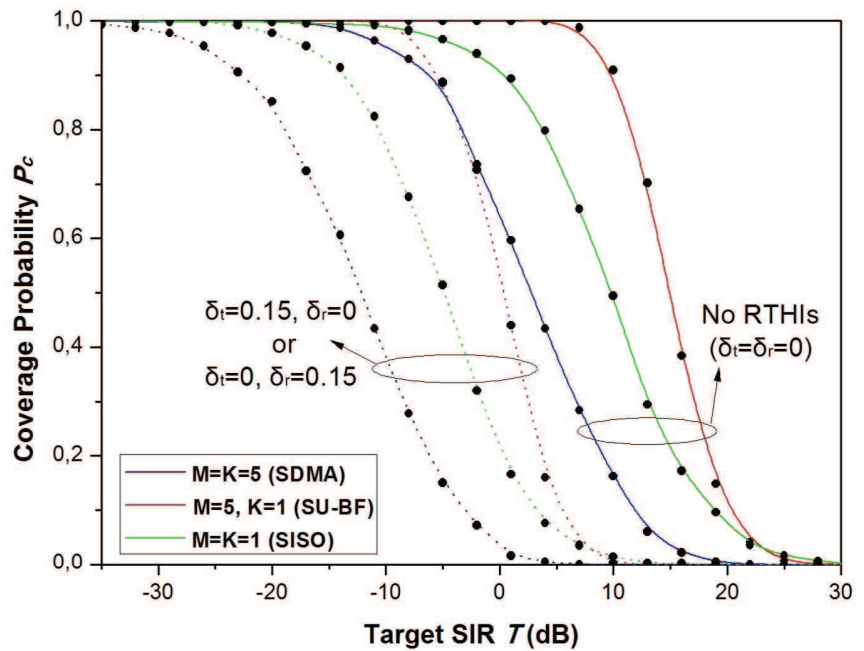

Fig. 1. Coverage probability versus the target SIR $T$ for varying ARTHIs and various transmission techniques $\left(\alpha=3, \lambda_{B}=3, \lambda_{u}=6 \lambda_{B}, p=23 \mathrm{~dB}\right.$.)

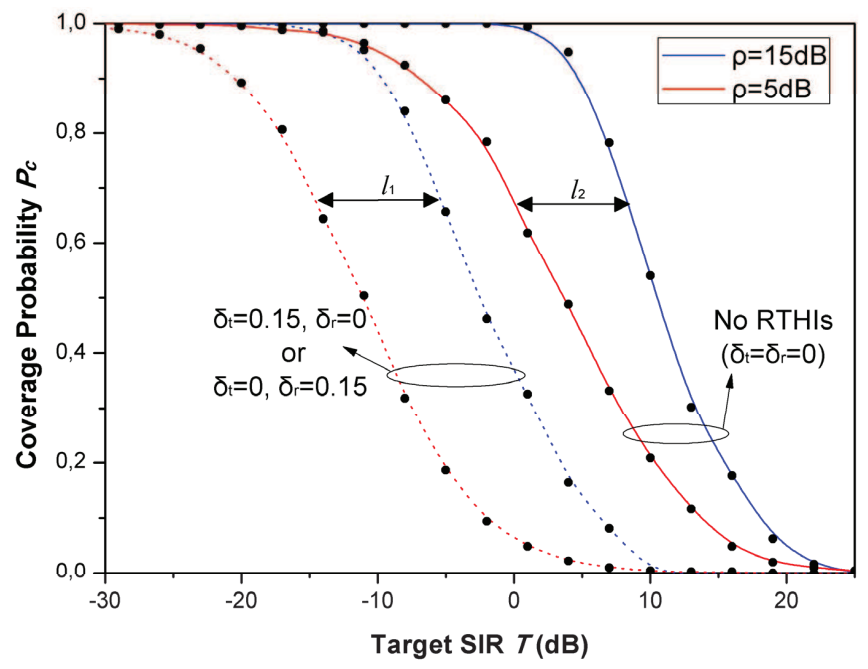

Fig. 2. Coverage probability versus the target SIR $T$ for varying ARTHIs and various transmit powers $\left(\alpha=3, \lambda_{B}=3, \lambda_{u}=6 \lambda_{B}\right.$.

columns $\overline{\mathbf{h}}_{k}=\frac{\mathbf{h}_{k}}{\left\|\mathbf{h}_{k}\right\|}$. In such case, the desired signal power, given by $h_{k}=\left|\mathbf{h}_{k}^{\mathrm{H}} \mathbf{w}_{k, k}\right| \cdot\left\|\mathbf{h}_{k}\right\|^{2}$, is $\Gamma(\Delta, 1)$ distributed with $\Delta=M-K+1$, since it equals to the product of two independent random variables distributed as $B(M-K+1, K-1)$ and $\Gamma(M, 1)$, respectively [17]. $I_{\boldsymbol{\eta}_{\mathrm{t}}}$ is obtained after taking the expectation over the transmit distorion noise of the tagged BS $I_{\eta_{\mathrm{t}}}=p_{k} \delta_{\mathrm{t}}^{2}\left\|\mathbf{h}_{k}\right\|^{2}$, which follows a scaled $\Gamma(M, 1)$ distribution. A similar result is obtained after taking the expectation over the receive distorion noise, i.e., $I_{\eta_{\mathrm{r}}}=p_{k} \delta_{\mathrm{r}}^{2}\left\|\mathbf{h}_{k}\right\|^{2}$, The other term in the denominator, concerning the interference from other BSs, $I_{l}$, is expressed in terms of the sum of two independent gamma distributed random variables $g_{l}=$ $\left|\mathbf{g}_{l}^{\mathrm{H}} \mathbf{s}_{l}\right|^{2} \sim \Gamma(K, 1)$. Note that $g_{l}$ is a $\Gamma(K, 1)$ random variable because the precoding matrices $\mathbf{W}_{l}$ coming from other BSs have unit-norm and are independent from the normalized $\overline{\mathbf{g}}_{l}$. Therefore, $g_{l}=\overline{\mathbf{g}}_{l}^{\mathrm{H}} \mathbf{W}_{l}$ is a linear combination of $K$ complex normal random variables, i.e., $g_{l} \sim \Gamma(K, 1)$. 


\section{APPENDIX B \\ PROOF OF THEOREM 1}

According to the definition of $p_{c}\left(T, \lambda_{B}, \alpha, \delta_{\mathrm{t}}, \delta_{\mathrm{r}}\right)$ and by means of appropriate substitution of the SIR $\gamma_{k}$, we have

$$
\begin{aligned}
& p_{c}\left(T, \lambda_{B}, \alpha, \delta_{\mathrm{t}}, \delta_{\mathrm{r}}\right)=\mathbb{E}\left[\mathbb{1}\left(\underset{x \in \Phi_{B}}{\cup} \operatorname{SIR}(x)>T\right)\right] \\
& \leq \mathbb{E}\left[\underset{x \in \Phi_{B}}{\cup} \mathbb{1}(\mathrm{SIR})>T\right] \\
& =\mathbb{E}\left[\sum_{x \in \Phi_{B}} \mathbb{P}[\mathrm{SIR}>T \mid l]\right] \\
& =\lambda_{B} \int_{x \in \mathbb{R}^{2}} \mathbb{E}\left[\mathbb{P}\left[h_{k}>\tilde{T}\left(I_{\boldsymbol{\eta}_{\mathrm{t}}}+I_{\boldsymbol{\eta}_{\mathrm{r}}}\right)+\tilde{T} l^{\alpha} I_{l} \mid l\right]\right] \mathrm{d} x,
\end{aligned}
$$

where in (12), we have used the Campbell-Mecke Theorem [30]. Given that $h_{k}$ is gamma distributed, i.e., $h_{k} \stackrel{\mathrm{d}}{\sim} \Gamma\left(\Delta_{k}, 1\right)$, we have $\mathbb{P}_{h_{k}}(z)=e^{-z} \sum_{i=0}^{\Delta-1} \frac{z^{i}}{i !}$. Thus, the integrable part of (13) can be written as

$$
\begin{aligned}
& \mathbb{P}\left[h_{k}>\tilde{T}\left(I_{\boldsymbol{\eta}_{\mathrm{t}}}+I_{\boldsymbol{\eta}_{\mathrm{r}}}\right)+\tilde{T} l^{\alpha} I_{l} \mid l\right]=e^{-\left(\tilde{T}\left(I_{\boldsymbol{\eta}_{\mathrm{t}}}+I_{\boldsymbol{\eta}_{\mathrm{r}}}\right)+\tilde{T} l^{\alpha} I_{l}\right)} \\
& \times \sum_{i=0}^{\Delta-1} \sum_{k=0}^{i}\left(\begin{array}{l}
i \\
k
\end{array}\right) \frac{\left(\tilde{T}\left(I_{\boldsymbol{\eta}_{\mathrm{t}}}+I_{\boldsymbol{\eta}_{\mathrm{r}}}\right)\right)^{i-k}\left(\tilde{T} l^{\alpha} I_{l}\right)^{k}}{i !}
\end{aligned}
$$

where in (14), we have applied the binomial theorem. Taking the expectation, we obtain

$$
\begin{aligned}
& \mathbb{E}\left[\mathbb{P}\left[h_{k}>\tilde{T}\left(I_{\boldsymbol{\eta}_{\mathrm{t}}}+I_{\boldsymbol{\eta}_{\mathrm{r}}}\right)+\tilde{T} l^{\alpha} I_{l} \mid l\right]\right]=\sum_{i=0}^{\Delta-1} \sum_{k=0}^{i} \sum_{n=0}^{i-k}\left(\begin{array}{c}
i \\
k
\end{array}\right)\left(\begin{array}{c}
i-k \\
n
\end{array}\right) \\
& \times \frac{(-1)^{i} \tilde{T}^{i-k} s^{k}}{i !} \frac{\mathrm{d}^{n}}{\mathrm{~d} s^{n}} \mathcal{L}_{I_{\boldsymbol{\eta}_{\mathrm{r}}}}(s) \frac{\mathrm{d}^{i-k-n}}{\mathrm{~d} s^{i-k-n}} \mathcal{L}_{I_{\boldsymbol{\eta}_{\mathrm{t}}}}(s) \frac{\mathrm{d}^{k}}{\mathrm{~d} s^{k}} \mathcal{L}_{I_{l}}(s),
\end{aligned}
$$

where we have set $\tilde{T}=T p_{k}^{-1}$ and $s=\tilde{T} l^{\alpha}$. In 14 we have made use of the Binomial theorem, and in 115 we have used the definition of the Laplace Transform $\mathbb{E}_{I}\left[e^{-s I}(s I)^{i}\right]=s^{i} \mathcal{L}\left\{t^{i} g_{I}(t)\right\}(s)$ and the Laplace identity $t^{i} g_{I}(t) \longleftrightarrow(-1)^{i} \frac{\mathrm{d}^{n}}{\mathrm{~d}^{n} s} \mathcal{L}_{I}\left\{g_{I}(t)\right\}(s)$. The Laplace transform $\mathcal{L}_{I_{l}}(s)$ is obtained by means of Proposition 2, while $\mathcal{L}_{I_{\eta_{\mathrm{t}}}}$ and $\mathcal{L}_{I_{\eta_{\mathrm{r}}}}(s)$ are provided by Lemma 1 Substitution of (15) into 13 concludes the proof.

\section{APPENDIX C \\ PROOF OF PROPOSITION 2}

Having defined $g_{l}$, accounting for the interference channel coefficient, which has identical distribution for all $l$, and for the transmit channel impairments from other BSs, the Laplace transform of the interference part $\mathcal{L}_{I_{l}}(s)$ can be derived as

$$
\begin{aligned}
& \mathcal{L}_{I_{l}}(s)=\mathbb{E}_{I_{l}}\left[e^{-s I_{l}}\right]=\mathbb{E}_{I_{l}}\left[e^{-s \sum_{l \in \Phi_{B} \backslash x} p_{l} g_{l} y^{-\alpha}}\right] \\
& =\mathbb{E}_{\Phi_{B}, g_{l}}\left[\prod_{l \in \Phi_{B} \backslash x} e^{-s p_{l} g_{l} y^{-a}}\right] \\
& =\mathbb{E}_{\Phi_{B}}\left[\prod_{l \in \Phi_{B} \backslash x} \mathcal{L}_{g_{l}}\left(s p_{l} y^{-a}\right)\right] \\
& =\exp \left(-\lambda_{B} \int_{\mathbb{R}^{2}}\left(1-\mathcal{L}_{g_{l}}\left(s p_{l} y^{-a}\right)\right) \mathrm{d} y\right)
\end{aligned}
$$

$$
\begin{aligned}
& =\exp \left(-2 \pi \lambda_{B} \int_{0}^{\infty}\left(\frac{\sum_{m=1}^{K}\left(\begin{array}{l}
K \\
m
\end{array}\right)\left(s p_{l} r^{-a}\right)^{m}}{\left(1+s p_{l} r^{-a}\right)^{K}} r \mathrm{~d} r\right)\right. \\
& =\exp \left(-\frac{2 \pi \lambda_{B} p_{l^{\frac{2}{a}}} s^{\frac{2}{a}}}{\alpha} \sum_{m=1}^{K}\left(\begin{array}{l}
K \\
m
\end{array}\right) \mathrm{B}\left(K-m+\frac{2}{a}, m-\frac{2}{a}\right)\right),
\end{aligned}
$$

where $\mathrm{B}\left(K-m+\frac{2}{a}, m-\frac{2}{a}\right)$ is the Beta function defined in [18, Eq. (8.380.1)]. Note that (16) comes from the independence among the locations of the BSs, while (16) holds due to the independence between the spatial and the fading distributions. Using the property of PGFL of PPP [19], we obtain (18), and the next step follows by substituting the Laplace transform of $g_{l}$, obtained in Lemma 2. Application of the Binomial theorem in (18), and conversion of the Cartesian coordinates to polar coordinates results to (19). The proof is concluded with the calculation of the integral. Specifically, it is obtained after substitution of $\left(1+r^{-\alpha}\right)^{-1} \rightarrow t$, many algebraic manipulations, and the use of [18, Eq. (8.380.1)].

\section{REFERENCES}

[1] A. Osseiran and et al., "Scenarios for 5G mobile and wireless communications: The vision of the METIS project," IEEE Commun. Mag. vol. 52, no. 5, pp. 26-35, May 2014.

[2] J. G. Andrews, F. Baccelli, and R. K. Ganti, "A tractable approach to coverage and rate in cellular networks," IEEE Trans. on Commun. vol. 59, no. 11, pp. 3122-3134, 2011.

[3] M. Kountouris and J. G. Andrews, "Downlink SDMA with limited feedback in interference-limited wireless networks," IEEE Trans. on Wireless Commun., vol. 11, no. 8, pp. 2730-2741, 2012

[4] H. S. Dhillon, M. Kountouris, and J. G. Andrews, "Downlink MIMO HetNets: Modeling, ordering results and performance analysis," IEEE Trans. on Wireless Commun., vol. 12, no. 10, pp. 5208-5222, 2013.

[5] A. Papazafeiropoulos, "Impact of general channel aging conditions on the downlink performance of massive MIMO," accepted in IEEE Trans. on Veh. Tech., 2016. [Online]. Available: http://arxiv.org/abs/1605.07661

[6] J. Qi and S. Aïssa, "On the power amplifier nonlinearity in MIMO transmit beamforming systems," IEEE Trans. Commun., vol. 60, no. 3, pp. 876-887, 2012.

[7] $\_$, "Analysis and compensation of I/Q imbalance in MIMO transmitreceive diversity systems," IEEE Trans. Commun., vol. 58, no. 5, pp. 1546-1556, 2010

[8] C. Studer, M. Wenk, and A. Burg, "MIMO transmission with residual transmit-RF impairments," in ITG/IEEE Work. Smart Ant. (WSA). IEEE, 2010, pp. 189-196.

[9] E. Björnson, P. Zetterberg, M. Bengtsson, and B. Ottersten, "Capacity limits and multiplexing gains of MIMO channels with transceive impairments," IEEE Commun. Lett., vol. 17, no. 1, pp. 91-94, 2013.

[10] E. Björnson, J. Hoydis, M. Kountouris, and M. Debbah, "Massive MIMO systems with non-ideal hardware: Energy efficiency, estimation, and capacity limits," IEEE Trans. Inform. Theory, vol. 60, no. 11, pp 7112-7139, Nov 2014.

[11] E. Björnson, M. Matthaiou, and M. Debbah, "Massive MIMO with nonideal arbitrary arrays: Hardware scaling laws and circuit-aware design,' IEEE Trans. Wireless Commun., vol. 14, no. no.8, pp. 4353-4368, Aug. 2015.

[12] A. K. Papazafeiropoulos, S. K. Sharma, and S. Chatzinotas, "Impact of transceiver impairments on the capacity of dual-hop relay massive MIMO systems," in 2015 IEEE Globecom Workshops (GC Wkshps), Dec 2015, pp. 1-6.

[13] A. Papazafeiropoulos, S. K. Sharma, and S. Chatzinotas, "MMSE filtering performance of DH-AF massive MIMO relay systems with residual transceiver impairments," in IEEE International Conference on Communications (ICC 2016), Kuala Lumpur, Malaysia, May 2016.

[14] H. Huang, C. B. Papadias, and S. Venkatesan, MIMO Communication for Cellular Networks. Springer Science \& Business Media, 2011.

[15] T. Schenk, RF imperfections in high-rate wireless systems: impact and digital compensation. Springer Science \& Business Media, 2008.

[16] H. Holma and A. Toskala, LTE for UMTS: Evolution to LTE-Advanced, Wiley, Ed., 2011.

[17] N. Jindal, "MIMO broadcast channels with finite-rate feedback," IEEE Trans. on Inform. Theory, vol. 52, no. 11, pp. 5045-5060, 2006.

[18] I. S. Gradshteyn and I. M. Ryzhik, "Table of integrals, series, and products," Alan Jeffrey and Daniel Zwillinger (eds.), Seventh edition (Feb 2007), vol. 885, 2007.

[19] S. N. Chiu, D. Stoyan, W. S. Kendall, and J. Mecke, Stochastic geometry and its applications. John Wiley \& Sons, 2013. 